\title{
Influence of repeated painful procedures and sucrose analgesia on the development of hyperalgesia in newborn infants
}

\author{
Anna Taddio ${ }^{a, b, *}$, Vibhuti Shah ${ }^{c, d}$, Eshetu Atenafu ${ }^{b}$, Joel Katz ${ }^{\text {d,e,f }}$ \\ a Pharmacy Practice Division, Leslie Dan Faculty of Pharmacy, University of Toronto, \\ ${ }^{b}$ Child Health Evaluative Sciences, The Hospital for Sick Children, Toronto, Canada \\ 'Dept. of Paediatrics, Mount Sinai Hospital, Toronto, Canada \\ ${ }^{\mathrm{d}}$ Faculty of Medicine, University of Toronto, Toronto, Canada \\ e Dept. of Psychology, York University, Toronto, Canada \\ ${ }^{\mathrm{f}}$ Dept, of Anaesthesia, Mount Sinai Hospital, Toronto, Canada
}

\begin{abstract}
A B S T R A C T
This study determined the effects of cumulative exposure to painful needle procedures and sucrose analgesia on the development of remote hyperalgesia in newborn infants, defined as an increase in response to a normally painful stimulus at a site distal from the site of injury. One-hundred and twenty healthy newborns and 120 healthy newborn infants of diabetic mothers equally randomized to sucrose analgesia or placebo prior to all needle procedures in the first two days after birth were divided into two exposure groups according to number of needle procedures they had undergone [high $(\geqslant 5)$ or low $(\leqslant 4)$ ] using the median cut-off technique. Compared to the low exposure group, infants in the high exposure group had a higher pain response during a subsequent venipuncture distal to the site of previous injury, assessed by the Premature Infant Pain Profile (PIPP) [7.1 vs. 8.4; $p=0.012]$ and Visual Analog Scale (VAS) $[2.5 \mathrm{~cm}$ vs. $3.2 \mathrm{~cm} ; p=0.047$, and a trend for longer cry duration [25.7 s vs. $33.8 \mathrm{~s} ; p=0.171$ ]. PIPP scores did not differ during a routine diaper change, suggesting a nociceptive specific mechanism for the remote hyperalgesia to venipuncture. Sucrose reduced PIPP, VAS, and cry duration scores during venipuncture, but did not prevent hyperalgesia $(p>0.05)$. There was a preponderance of infants of diabetic mothers in the high exposure group; however, the analysis did not demonstrate this to be a confounding factor. In conclusion, sucrose analgesia for repeated painful procedures in the first day of life does not prevent development of remote hyperalgesia in newborns.
\end{abstract}

\section{Introduction}

Newborn infants are subjected to procedural pain as part of routine medical care. Untreated procedural pain can lead to profound adverse effects. We previously demonstrated that newborn infants born to mothers with diabetes and who underwent repeated heel lances in the first 24-36 h of life exhibited hyperalgesia (an increased response to a stimulus which is normally painful) during a subsequent venipuncture compared to normal infants who had not undergone repeated painful procedures [25]. Other studies have demonstrated that injury during infancy has local and global consequences on sensory processing that can be observed years later [16].

A significant percentage of newborn infants are subjected to repeated procedural pain. Infants of diabetic mothers (IDMs) alone constitute approximately $5 \%$ of live births [18]. An additional 10$15 \%$ of newborns require prolonged hospitalization after delivery

\footnotetext{
* Corresponding author. Tel.: +416 978 8822; fax: +416978 1833.

E-mail address: anna.taddio@utoronto.ca (A. Taddio).
}

for medical reasons including prematurity, congenital anomalies, sepsis, hyperbilirubinemia and feeding difficulties [13]. Individually, these infants may be exposed to up to hundreds of painful procedures [9]. Reducing the burden of pain from medical procedures in newborn infants is therefore clinically important $[4,5]$.

Sucrose (table sugar) is a naturally occurring sweetener with analgesic effects in young infants. Although not fully understood, the mechanism of action is postulated to involve activation of the endogenous opioid system through taste [7]. This is supported by the presence of opioid receptors on the tongue and animal studies demonstrating opioid-antagonist reversible analgesia during noxious stimulation [19]. Moreover, chronic consumption of sweeteners has been shown to negate the analgesic effects of opioids [14].

Sucrose has been promoted for analgesia in clinical practice guidelines and consensus statements based on systematic reviews demonstrating its efficacy for common medical procedures such as heel lance and venipuncture $[2,3,8,21,22]$. There is a lack of data, however, on the effects of repeated doses. Specifically, it is unclear 
whether consistent management of pain with sucrose prevents the development of exaggerated pain responses in the future in infants who undergo repeated procedures.

This study was undertaken to determine the potential effects of: (i) cumulative exposure to painful medical procedures, and (ii) consistent management of procedural pain with sucrose, on the development of remote hyperalgesia in newborn infants in the first two days of life. The specific objectives were to determine the-effects of exposure to a high vs. low number of painful procedures and administration of sucrose vs. placebo on the subsequent development of remote hyperalgesia, defined as an increase in response to a venipuncture performed in uninjured skin on the dorsum of the hand.

\section{Materials and methods}

We studied newborn infants $\geqslant 36$ weeks gestational age at birth that were enrolled in a double-blind randomized controlled trial of the effectiveness of oral sucrose administered prior to every noxious medical procedure performed after birth [26]. In order to ensure inclusion of infants undergoing a wide range in number of medical procedures, we targeted normal newborn infants (born of women with uneventful pregnancies) and IDMs. Normal newborn infants usually undergo one medical procedure in the first day of life (intramuscular injection of vitamin $\mathrm{K}$ ), with additional procedures undertaken as medically necessary. IDMs, on the other hand, routinely undergo at least 3 (and upwards of 10 or more) heel lances in addition to vitamin $\mathrm{K}$ injection in the first day of life to monitor blood glucose levels [29]. Other than the tendency to develop hypoglycemia, which is promptly treated when diagnosed, these infants are considered healthy and room-in with their mothers on the postnatal ward. We excluded infants that were admitted to the neonatal intensive care unit, scheduled to undergo circumcision during the study period, had a major congenital or neurological anomaly, had a clinical diagnosis of birth asphyxia [1] or seizures, or were receiving analgesics or sedatives.

All infants were randomized to receive either $2 \mathrm{ml}$ of $24 \%$ sucrose (weight/volume) or $2 \mathrm{ml}$ of sterile water (placebo) by mouth 2 minutes prior to all tissue-damaging needle procedures performed from birth, including the newborn screening test, performed by venipuncture on the dorsum of the hand, approximately $24 \mathrm{~h}$ later. The randomization sequence generation and concealment of allocation details have been previously published [26].

The study was approved by the Research Ethics Boards of Mount Sinai Hospital, The Hospital for Sick Children, York University and Health Canada, and was conducted on the Mother and Baby Unit at Mount Sinai Hospital, Toronto, Canada.

The newborn screening test was performed using a venipuncture on the dorsum of the hand rather than a heel lance (the usual method of blood sampling in newborns in the study centre). This approach enabled us to assess the presence of hyperalgesia in a remote, uninjured region of the body (i.e., hand) subsequent to repeated injury elsewhere on the body. Thus, we tested the hypothesis that repeated injury would induce a remote hyperalgesia to non-inflamed, non-sensitized skin in the hand. We defined hyperalgesia as an increase in response to venipuncture for infants exposed to a high number of needle procedures when compared to a control group of infants exposed to a low number of procedures. Venipuncture is also less painful than heel lance [17] and so was selected to minimize pain in all participants.

All data were collected prospectively in a standardized method previously described [26]. Infant pain responses were video recorded during the first tissue-damaging needle procedure after delivery (intramuscular vitamin $\mathrm{K}$ injection administered on the anterior aspect of the thigh, within the first hour of life) and venipuncture for the newborn screening test (administered approximately $24 \mathrm{~h}$ later on the dorsum of the hand). In addition, infant responses were video recorded during a non-tissuedamaging handling procedure (i.e., diaper change) performed within the first hours of life and again after the newborn screening test. Approximately $5 \mathrm{~min}$ prior to each procedure, a portable pulse oximeter (Datex-Ohmeda 3900P) was applied to the-infant's foot and a digital camera (Sony DCRTRV25) and tripod were positioned approximately $1 \mathrm{~m}$ from the infant's body. The infant's face was videotaped during the entire procedure. Physiological (heart rate and oxygen saturation) responses were also recorded continuously during the procedure. All procedures were carried out by certified nurse(s) or physician(s) as per standard clinical practice.

\subsection{Pain outcome measures}

We assessed the infants' pain responses to vitamin $\mathrm{K}$ injection and venipuncture using three validated measures of pain: the Premature Infant Pain Profile (PIPP) [20], an observer-rated, global behavioural measure using a $10 \mathrm{~cm}$ visual analog scale (VAS) for pain, and cry duration [25]. We assessed infants' responses to diaper change using the PIPP.

As previously described [26], the primary outcome was the PIPP score. The PIPP was developed from pain responses observed in preterm infants and subsequently validated in preterm and full-term infants [21]. It incorporates three behavioural (facial actions: brow bulge, eyes squeezed shut, naso-labial furrow), two physiological (heart rate, oxygen saturation) and two contextual (gestational age, infant state) indicators of pain that are scored individually on a 4-point scale from 0 to 3 and then summed together for an overall score. A research assistant that was unaware of treatment allocation, exposure group and study hypotheses, scored each facial action as present or absent in 2-s intervals from the videotaped footage for the first $30 \mathrm{~s}$ of the venipuncture (first $20 \mathrm{~s}$ of intramuscular injection of vitamin $\mathrm{K}$, or less if the procedure lasted less than $20 \mathrm{~s}$ ) using established methods [21]. The percentage of the total time that each facial action was observed during each phase was calculated and used to assign a score of $0-3$. For physiologic data, heart rate and oxygen saturation change from baseline was recorded over the same time frame and used to assign a score of 0-3. A total PIPP score was computed for each phase of the procedure by summing the scores of the seven indicators. In full-term infants, PIPP scores can vary from 0 (no pain) to 18 (maximum pain) [21]. At present, the PIPP is the most commonly used multidimensional measure of pain in infants and incorporates the indicators of pain most frequently used.

Secondary measures of pain included a $10 \mathrm{~cm}$ VAS for pain with endpoints labelled "no pain" and "worst possible pain", and total crying time in seconds, defined as audible sound in the presence of facial grimacing. VAS pain scores and cry duration were independently scored from the videotapes by two other research assistants who were also unaware of treatment allocation, exposure group, and study hypotheses.

\subsection{Statistical analysis}

Using Power Analysis and Statistical Software, PASS (NCSS Statistical Software, Kaysville, Utah), our sample size calculation determined that 240 infants provided $95 \%$ power to detect a difference of $20 \%$ between high and low exposure groups with an interaction between exposure and drug treatment groups. For this analysis, infants were divided into two exposure groups, using 
Table 1

Characteristics of participating newborn infants.

\begin{tabular}{|c|c|c|c|}
\hline & Exposure group & Het & \multirow{2}{*}{ Value } \\
\hline & $\begin{array}{l}\text { Low exposure ( } 54 \\
\text { painful procedures) } \\
n=133\end{array}$ & $\begin{array}{l}\text { High exposule }(\geqslant 5 \\
\text { painful procedures) } \\
n=81\end{array}$ & \\
\hline \\
\hline $\begin{array}{l}\text { Meant }(\mathrm{SD}) \\
\text { gestational age } \\
\text { (weeks) }\end{array}$ & $39.1(1)$ & $39(13)$ & 0.378 \\
\hline $\begin{array}{l}\text { Mean (SD) birth } \\
\text { weight (Kg) }\end{array}$ & $3.4(0.4)$ & $3.5(0.5)$ & 0.222 \\
\hline No. of males (\%) & $59(44)$ & $47(58)$ & 0.052 \\
\hline $\begin{array}{l}\text { No, vaginal delivery } \\
\text { (\%) }\end{array}$ & $58(44)$ & $33(41)$ & 0.681 \\
\hline $\begin{array}{l}\text { No. randomized to } \\
\text { sucrose }(\%)\end{array}$ & $68(51)$ & $40(49)$ & 0.804 \\
\hline \multicolumn{4}{|c|}{ Pain response during intramuscular injection of vitamin $K$} \\
\hline $\begin{array}{l}\text { Mean }(\mathrm{SD}) \\
\text { premature infant } \\
\text { pain profile }\end{array}$ & $7.7(3.5)$ & $69(3.8)$ & 0.131 \\
\hline $\begin{array}{l}\text { Mean (SD) visual } \\
\text { analog scale }(\mathrm{cm})\end{array}$ & $3.9(2.7)$ & $3.7(2.6)$ & 0.734 \\
\hline $\begin{array}{c}\text { Mean (SD) cry } \\
\text { duration }\end{array}$ & $2.3(3.6)$ & $22(2.9)$ & 0755 \\
\hline
\end{tabular}

Values are means (standard deviation, SD) or frequency (percent).

${ }^{*} n=131$ for Premature Infant Pain Profile in infants in low exposure ( $\leqslant 4$ painful procedures) group and $n=79$ for Premature Infant Pain Profile in infants in high exposure ( $\geqslant 5$ painful procedures) group.

** Student's $t$-test or $\chi^{2}$ test, as appropriate

the median cut-off technique, based on the number of painful procedures they had undergone [high $(\geqslant 5)$ or low $(\leqslant 4)$ ] prior to the newborn screening test. Univariate 2-factor ANCOVAs were used to compare PIPP scores, VAS pain and cry duration scores during the newborn screening test between exposure groups (high vs. low number of procedures) and drug treatment groups (sucrose vs. placebo), adjusting for the baseline pain score during vitamin $\mathrm{K}$ injection.

The number of painful procedures that infants had undergone prior to venipuncture was correlated with PIPP scores, VAS pain scores and cry duration during venipuncture (after subtracting baseline scores during vitamin $\mathrm{K}$ injection) using Pearson's $r$. Demographic data and baseline PIPP scores, VAS pain scores and cry duration scores during vitamin $\mathrm{K}$ injection were analyzed using $\chi^{2}$ test, or Student's $t$-test, as appropriate.

In order to rule out the possibility that observed differences between exposure groups in the pain outcome measures were due to underlying maternal medical condition (IDMs vs. normal infant) we compared PIPP scores, VAS pain scores and cry duration between IDMs and normal infants, adjusting for analgesic treatment allocation and number of painful procedures.
In order to determine whether exposure-related increases in pain responses were specific to noxious (tissue-damaging) stimuli or generalized to non-noxious stimuli as well, we compared infant PIPP scores during a diaper change performed after the venipuncture using a 2-factor (exposure group and drug treatment group) ANCOVA, adjusting for baseline diaper change PIPP scores obtained within the first few hours after birth.

An intent-to-treat approach was used that included all infants where outcome data were obtained. A $p$ value of 0.05 or less was considered statistically significant. Data were analyzed using SAS (version 9.1; SAS Institute Inc., Cary, NC).

\section{Results}

Of 240 infants enrolled in the trial [26], outcome data were available for $214(89 \%)$ of infants. The remainder $(n=26)$ were excluded due to the following circumstances: clinical deterioration ( $n=11$ ), withdrawal of parental consent $(N=6)$, procedure missed $(n=5)$, major protocol violation $(n=3)$, and reason not documented $(n=1)$.

Using the median cut-off of $\mathbf{4}$ for the number of procedures experienced prior to venipuncture for the newborn screening test, 133 infants were assigned to the low exposure group ( $\leqslant 4$ prior procedures) and 81 were assigned to the high exposure group $(\geqslant 5$ prior procedures). Twenty-seven $(20 \%)$ of infants in the low exposure group, and 80 (99\%) of infants in the high exposure group were IDMs.

Demographic characteristics are shown in Table 1. Infant PIPP scores, VAS pain scores and cry duration during vitamin K injection (the first needle procedure performed after birth) did not differ between groups, indicating that infants were comparable at the very first painful procedure. The median (range) number of procedures for infants in the low and high exposure groups was $1(1-4)$ and 6 (5-13), respectively; $p<0.001$. Fig. 1 shows a schematic flow diagram of the timing and frequency of needle procedures in the low and high exposure groups.

\subsection{Pain response during venipuncture for the newborn screening test}

Compared to infants in the low exposure group, infants in the high exposure group had higher PIPP scores [mean (95\% Cl): 7.1 (6.5-7.8) vs. $8.4(7.6-9.2) ; p=0.012]$ and VAS pain scores $[2.5 \mathrm{~cm}$ $(2.1-2.9)$ vs. $3.2 \mathrm{~cm}(2.7-3.7) ; p=0.047]$ but not cry duration scores [25.7 s (18.6-32.9) vs. $33.8 \mathrm{~s} \quad(24.7-43.0) ; p=0.171]$ (Fig. 2). Pre-treatment with sucrose resulted in lower PIPP [mean $(95 \% \mathrm{Cl}): 6.5(5.8-7.2)$ vs. $9.1(8.4-9.8) ; p<0.0001]$, VAS pain $[1.9$ $(1.4-2.4)$ vs. $3.8(3.3-4.3) ; p<0.0001]$, and cry duration [13.1 (5.0-21.2) vs. $46.3(38.2-54.4) ; p<0.0001]$ scores (Fig. 2). There were no statistically significant interactions between procedure exposure level and treatment allocation ( $p=0.742$ for PIPP,

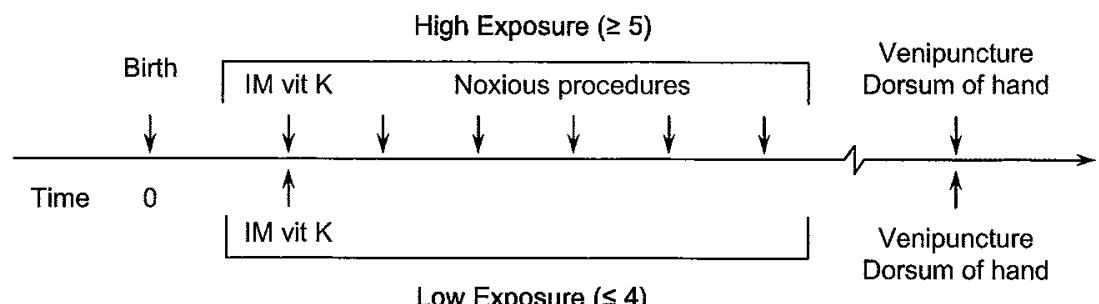

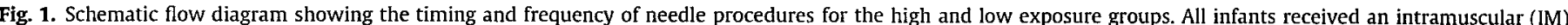

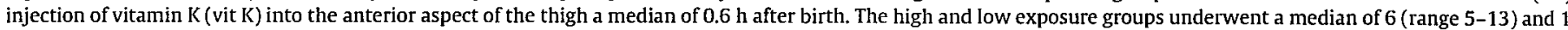

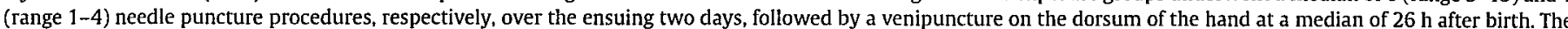

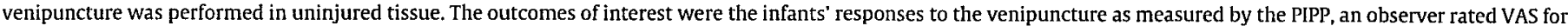
pain, and cry duration. 


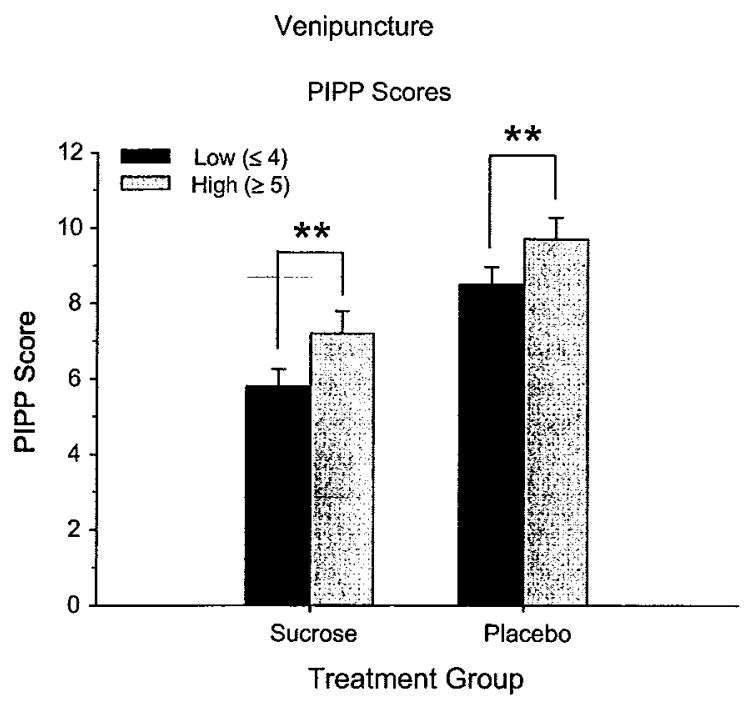

VAS Pain Scores

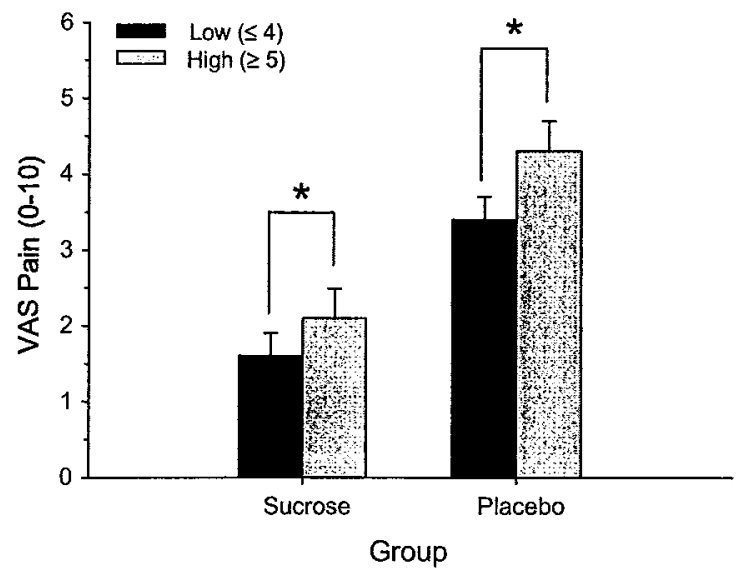

Cry Duration

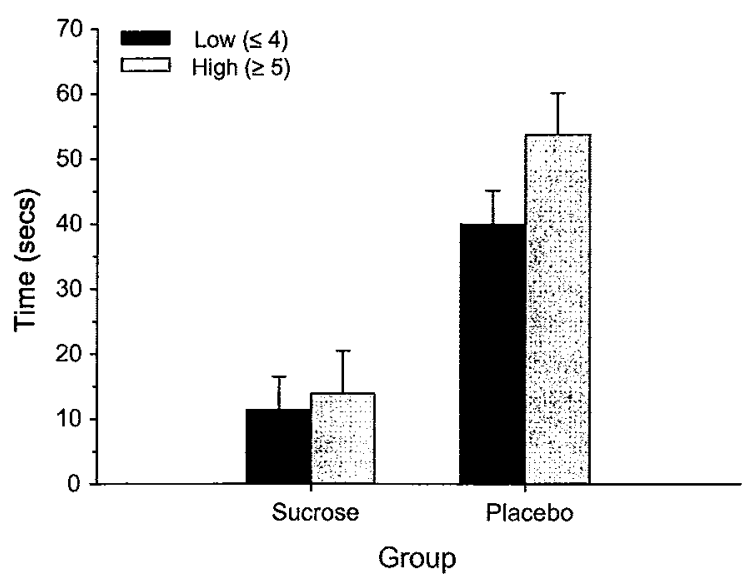

Fig. 2. Pain responses during Venipuncture for the newborn screening test values are means (standard error, SE). ANCOVA results: "*PIPP (Premature Infant Pain Profile) scores: $p=0.012$ for exposure group; $p<0.0001$ for treatment group. "VAS (Visual Analog Scale) pain scores: $p=0.047$ for exposure group; $p<0.0001$ for treatment group. Cry duration: $p=0.171$ for exposure group; $p<0.0001$ for treatment group. For all analyses, $n=133$ in low exposure ( $\leqslant 4$ painful procedures) group and $n=81$ in high exposure ( $\geqslant 5$ painful procedures) group; except for PIPP scores where $n=130$ in low exposure group and $n=79$ in high exposure group.

$p=0.547$ for VAS pain scores, and $p=0.344$ for cry duration, respectively).
The number of painful procedures was correlated with PIPP scores (Pearson's $r=0.2, p=0.004)$ and VAS pain scores $(r=0.14$, $p=0.044$ ), but not cry duration $(r=0.08, p=0.238$ ).

Secondary analyses comparing pain responses between IDMs and normal infants, adjusting for number of painful procedures and analgesic treatment allocation, did not demonstrate any differences for any of the outcome measures ( $p=0.965$ for PIPP scores, $p=0.277$ for VAS pain scores, and $p=0.365$ for cry duration). In addition, there were no significant differences in pain responses between the IDMs and normal infants within the low exposure group ( $p>0.05$ for all analyses).

Secondary analyses comparing PIPP scores in response to a diaper change after the venipuncture did not show a significant difference between the high and low exposure groups nor a significant interaction between exposure and treatment (sucrose vs. placebo) groups $(p>0.05$ for all analyses). Infants treated with sucrose, however, had lower PIPP scores than infants treated with placebo $(p<0.001)[27]$.

\section{Discussion}

The present study demonstrates that infants exposed to a high number ( 5 or more) of needle puncture procedures in the first day of life display greater pain responses, as assessed using PIPP and VAS pain scores, during a subsequent venipuncture performed on the second day of life when compared with infants exposed to a low number of procedures. Administration of sucrose prior to all painful procedures reduced scores during the venipuncture at $24 \mathrm{~h}$. However, infants who experienced more frequent procedures had higher scores despite pre-treatment with sucrose.

This is the first study to evaluate the combined effects of cumulative exposure to tissue-damaging procedures and repeated sucrose administration on remote hyperalgesia in full-term infants. The results are consistent with our previous study demonstrating that IDMs have a heightened pain response to venipuncture when compared to normal infants [25]. In that study, IDMs had undergone an average of 10 additional painful heel lance procedures compared to normal infants. In the present study, we demonstrated that five procedures are sufficient for newborn infants to develop hyperalgesia to a subsequent venipuncture. The threshold for inducing increased behavioural responsiveness to painful procedures in neonates is likely close to five procedures given that in a previous study, three heel lances failed to result in a noticeable change in pain behaviours over time although heart rate increased with successive procedures [15].

Two decades ago, in the first study of hyperalgesia in neonates, Fitzgerald et al. [10] demonstrated that flexor reflex thresholds in preterm infants were diminished in an area of inflamed skin due to previous tissue damage. Thresholds were not, however, altered in uninjured skin [10]. Subsequently, in the first study of remote hyperalgesia in full-term newborn infants, we demonstrated that male circumcision was associated with an increase in infant pain responses at 4- and 6-month routine immunization [23]. In a follow-up study, we showed that treatment of circumcision pain with topical local anaesthesia attenuated these increased responses, suggesting a protective effect of analgesia [24]. The observed ineffectiveness of sucrose in preventing hyperalgesia in the present study may be because it is a relatively weak analgesic, development of tolerance occurs after repeated administration, or hyperalgesia is mediated by processes that are not influenced by sucrose. In our effectiveness analysis of the present study, sucrose conferred only a modest (16\%) reduction in overall infant pain when used for all painful procedures [26], consistent with other recently published data [12]. It is possible that this magnitude of pain reduction is insufficient to prevent hyperalgesia from occurring, 
and analgesic strategies aimed at preventing rather than decreasing pain, are needed. The lack of effect of sucrose may also depend on the development of tolerance. However, there was no evidence of tolerance in our effectiveness analysis [26]. Finally, although the exact mechanisms by which sucrose exerts its analgesic effects are not known, it is presumed to activate the endogenous opioid system [7]. It is well established that opioids are relatively ineffective at reducing hyperalgesia associated with the $\mathrm{N}$-methyl-D-aspartate (NMDA)-mediated state of central sensitization that arises after injury [30].

The finding that PIPP scores in response to venipuncture, but not diaper change, were significantly greater in the high vs. low exposure group suggests that the mechanism underlying the remote hyperalgesia is nociceptive specific. Although we do not know the precise mechanism underlying the remote hyperalgesia, we suggest it may involve the absence of endogenous inhibitory controls in young infants in combination with the stress effects produced by the hypothalamo-pituitary-adrenocortical and the sympatho-adreno-medullary systems. It is possible that the stress response and sympathetic arousal associated with prior tissuedamaging procedures in the high exposure group induce a widespread sensitization to subsequent C-fiber strength noxious inputs. The immature descending inhibitory control system in the neonate would therefore not be capable of mounting an effective counter response [11] to the excitatory, noxious inputs associated with the venipuncture, leading to a (spatially) remote hyperalgesia in the high exposure group.

It is possible that higher PIPP and VAS pain scores observed in infants exposed to many vs. few procedures may be due to underlying differences in the infants (such as altered in-utero environment associated with the mother's diabetes status in IDMs). However, infants did not differ in their pain response during venipuncture as a function of maternal diabetes status (IDMs vs. normal) when the number of procedures experienced was accounted for, suggesting that it is cumulative exposure to procedures that underlies the development of hyperalgesia rather than the disease. Additional studies are needed, however, to rule out differences in pain responses due to underlying medical condition.

These data have implications for clinical practice since procedural pain is commonplace in hospitalized newborn infants [9], and sucrose has become widely adopted to manage procedural pain [28]. Based on the observed results, sucrose should not be given as the sole analgesic agent for the management of procedural pain in newborn infants undergoing repeated painful procedures. Additional research on the effects of multiple analgesic strategies combined together is needed.

There are several strengths of our study including inclusion of healthy full-term newborn infants which reduced the confounding effects of underlying medical condition or development on assessments of pain [6]; double-blind, randomized design for drug administration, minimizing bias; venipuncture performed in previously uninjured site thus avoiding the confounding effects of inflammation and hyperalgesia induced by repeated procedures [10]; and use of multiple validated measures of pain with a consistent pattern of results, increasing confidence in the interpretation. In addition, the study results are generalizable to a large infant population due to the inclusion of IDMs and normal infants, and the use of multiple nurses and physicians performing medical procedures.

In conclusion, newborn infants exposed to five or more tissuedamaging needle procedures over the first two days of life had higher pain responses, as assessed using the PIPP and VAS pain scores, during a subsequent venipuncture for the newborn screening test than infants exposed to four or fewer tissue-damaging needle procedures. Consistent use of sucrose for all procedures did not prevent the development of hyperalgesia to venipuncture. These results point to the need to: (i) minimize tissue-damaging needle procedures in the newborn period and, (ii) develop safe and effective agents to prevent pain and the development of hyperalgesia following multiple painful procedures.

\section{Prior presentation of these data}

This study was presented as a poster at the International Association for the Study of Pain (IASP) World Congress Meeting in Glasgow, Scotland on August 20, 2008.

\section{Funding}

The study was supported by a Canadian Institutes of Health Research (CIHR) grant (MCT 63143). In addition, the manufacturer of sucrose, Respironics Inc., provided the active and placebo supplies for the study. Dr. A. Taddio is supported by a New Investigator Award by CIHR. Dr. J. Katz is supported by a CIHR Canada Research Chair in Health Psychology at York University. The funding agencies did not provide any input regarding the design and conduct of the study; collection, management, analysis, and interpretation of the data; and preparation, review, or approval of the manuscript.

\section{Conflict of interest/disclosure}

\section{All authors have nothing to declare.}

\section{Author contributions}

Dr. Taddio conceived the study, designed the protocol, executed the study, provided support for the study, collected data, analyzed and interpreted the data, and wrote the manuscript. Dr. Taddio is the corresponding author and had final responsibility for the decision to submit for publication.

Dr. Shah conceived the study, designed the protocol, executed the study, collected data, and critically revised the manuscript with important intellectual content.

Dr. Katz conceived the study, designed the protocol, analyzed and interpreted the data, and critically revised the manuscript with important intellectual content.

Mr. Atenafu analyzed and interpreted the data, and critically revised the manuscript with important intellectual content.

\section{Acknowledgments}

We are grateful to the staff and parents of participating infants, for their support of this study and to Elizabeth Uleryk and Tamsin Adams-Webber for their assistance with manuscript preparation.

\section{References}

[1] American Academy of Paediatrics; American College of Obstetricians and Gynecologists. Relationships between perinatal factors and neurological outcome. In: Poland R, Freeman R, editors. Guidelines of Perinatal Care. 3rd ed. Elk Grove Village: American Academy of Pediatrics; 1992. p. 221-4.

[2] American Academy of Pediatrics, Canadian Paediatric Society. Prevention and management of pain and stress in the neonate. Pediatrics $2000 \cdot 105: 454-61$

[3] Anand KJ. Consensus statement for the prevention and management of pain in the newborn. Arch Pediatr Adolesc Med 2001;155:173-80.

[4] Anand KJ, Aranda JV, Berde CB, Buckman S, Capparelli EV, Carlo W, Hummel P. Johnston CC, Lantos J, Tutag-Lehr V, Lynn AM, Maxwell LG, Oberlander TF, Raju TN, Soriano SG, Taddio A, Walco GA. Summary proceedings from the neonatal pain-control group. Pediatrics 2006;117:S9-S22.

[5] Anand KJ, Johnston CC, Oberlander TF, Taddio A, Lehr VT, Walco GA. Analgesia and local anesthesia during invasive procedures in the neonate. Clin Ther 2005;27:844-76.

[6] Andrews K, Fitzgerald M. Biological barriers to paediatric pain management. Clin J Pain 1997:13:138-43.

[7] Blass EM, Watt LB. Suckling- and sucrose-induced analgesia in human newborns. Pain 1999;83:611-23. 
[8] Canadian Paediatric Society, American Academy of Pediatrics. Prevention and management of pain and stress in the neonate. Paediatr Child Health 2000;5:31-8.

[9] Carbajal R, Rousset A, Danan C, Coquery S, Nolent P, Ducrocq S, Saizou C, Lapillonne A, Granier M, Durand P, Lenclen R, Coursol A, Hubert P, de Saint Blanquat L, Boelle PY, Annequin D, Cimerman P, Anand $\mathrm{KJ}$, Breart $G$. Epidemiology and treatment of painful procedures in neonates in intensive care units. JAMA 2008;300:60-70.

[10] Fitzgerald M, Millard C, McIntosh N. Cutaneous hypersensitivity following peripheral tissue damage in newborn infants and its reversal with_topical anaesthesia. Pain 1989;39:31-6.

[11] Fitzgerald $M$, Walker SM. Infant pain management: a developmental neurobiological approach. Nat Clin Pract Neurol 2009;5:35-50.

[12] Gaspardo CM, Miyase CI, Chimello JT, Martinez FE, Martins Linhares MB. Is pain relief equally efficacious and free of side effects with repeated doses of oral sucrose in preterm neonates? Pain 2008;137:16-25.

[13] Health Canada, Perinatal health indicators for Canada: a resource manual. Minister of Public Works and Government Services Canada; 2000.

[14] Lieblich I, Cohen E, Ganchrow JR, Blass EM, Bergmann F. Morphine tolerance in genetically selected rats induced by chronically elevated saccharin intake. Science 1983;221:871-3.

[15] Pineles BL, Sandman CA, Waffarn F, Uy C, Davis EP. Sensitization of cardiac responses to pain in preterm infants. Neonatology 2007;91:190-5.

[16] Schmelzle-Lubiecki BM, Campbell KA, Howard RH, Franck L, Fitzgerald M. Long-term consequences of early infant injury and trauma upon somatosensory processing. Eur f Pain 2007;11:799-809.

[17] Shah VS, Taddio A, Bennett S, Speidel BD. Neonatal pain response to heel stick vs, venepuncture for routine blood sampling. Arch Dis Child Fetal Neonatal Ed 1997;77:F143-4.

[18] Shand AW, Bell JC, McElduff A, Morris J, Roberts CL. Outcomes of pregnancies in women with pre-gestational diabetes mellitus and gestational diabetes mellitus; a population-based study in New South Wales, Australia, 1998-2002. Diabet Med 2008;25:708-15.

[19] Shide DJ, Blass EM. Opioidlike effects of intraoral infusions of corn oil and polycose on stress reactions in 10-day-old rats. Behav Neurosci 1989;103:1168-75.

[20] Stevens B, Johnston C, Petryshen P, Taddio A. Premature Infant Pain Profile: development and initial validation. Clin J Pain 1996;12:13-22.

[21] Stevens B, Taddio A, Ohlsson A, Einarson T. The efficacy of sucrose for relieving procedural pain in neonates-a systematic review and meta-analysis. Acta Paediatr 1997;86:837-42.

[22] Stevens B, Yamada J. Ohlsson A. Sucrose for analgesia in newborn infants undergoing painful procedures. Cochrane Database Syst Rev 2004:CD001069.

[23] Taddio A, Goldbach M, lpp M, Stevens B, Koren G. Effect of neonatal circumcision on pain responses during vaccination in boys. Lancet 1995;345:291-2.

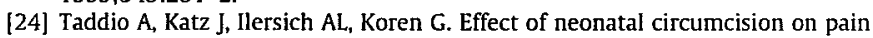
response during subsequent routine vaccination. Lancet 1997;349:599-603.

[25] Taddio A, Shah V, Gilbert-MacLeod C, Katz J. Conditioning and hyperalgesia in newborns exposed to repeated heel lances. JAMA 2002;288:857-61.

[26] Taddio A, Shah V, Hancock R, Smith RW, Stephens D, Atenafu E, Beyene J, Koren G, Stevens B, Katz J. Effectiveness of sucrose analgesia in newborns undergoing painful medical procedures. CMAJ 2008;179:37-43.

[27] Taddio A, Shah V, Katz J. Reduced infant response to a routine care procedure after sucrose analgesia. Pediatrics 2009;123:e425-9.

[28] Taddio A, Yiu A, Smith R, Katz J, McNair C, Shah V. Variability in clinical practice guidelines for sweetening agents in newborn infants undergoing painful procedures. Clin J Pain 2009;25:153-5.

[29] Williams AF. Hypoglycaemia of the newborn: a review. Bull World Health Organ 1997;75:261-90.

[30] Woolf CJ, Salter MW. Neuronal plasticity: increasing the gain in pain. Science 2000;288:1765-9. 